\title{
Worm Load and Septal Fibrosis of the Liver in Capillaria hepatica-infected Rats
}

\author{
Roseli Fernandes Oliveira, Zilton A Andrade ${ }^{+}$
}

\author{
Laboratório de Patologia Experimental, Centro de Pesquisas Gonçalo Moniz-Fiocruz, Rua Valdemar Falcão 121, \\ 40295-001 Salvador, BA, Brasil
}

Inocula, varying from 15 to 1,000 embryonated Capillaria hepatica eggs, were administered to young adult rats by gastric tube, in an attempt to investigate the influence of worm load in the production of septal fibrosis of the liver. Low doses of 15, 30 or 50 eggs were sufficient to produce septal fibrosis, but it appeared with variable degrees of intensity and always with focal distribution. Septal fibrosis became diffuse, progressive with time, and already well developed 40 days after infection, when 100 eggs or more were administered. However, higher inocula (200, 500 and 1,000 eggs) did not intensify septal fibrosis, although the number of parasitic focal lesions proportionally augmented.

Key words: Capillaria hepatica - septal hepatic fibrosis - worm load

Septal fibrosis is a frequent morphological type of hepatic fibrosis, represented by thin and straight fibrous tissue septa that dissect the liver parenchyma and sometimes circumscribe hepatocellular nodules in cirrhosis. Its pathogenesis is a matter of considerable interest (Bhunchet \& Waske 1992, Bhunchet \& Fujieda 1993, Onori et al. 2000). Septal fibrosis appears as the most prominent finding in a serious clinical condition of unknown etiology, named incomplete septal cirrhosis (Nevens et al. 1994, Dhar \& Chawla 1995). There are at least two experimental models for investigation of septal fibrosis of the liver, both in rats: the pig-serum model (Paronetto \& Popper 1966, Andrade 1991) and the Capillaria hepatica model (Ferreira \& Andrade 1993). This latter has advantages over the pig-serum model, since it is more rapid to develop (around 40 days) and appears regularly, in $100 \%$ of the infected rats. However, it is difficult to establish the worm load in a particular case, and so to correlate that variable in relation to septal fibrosis. Adult worms spontaneously die within the liver of rats 20-30 days after infection, and septal fibrosis starts soon after that (Ferreira \& Andrade 1993). Parasitic focal lesions may contain from one to several dead worms and the number of eggs in each lesion may

\footnotetext{
${ }^{+}$Corresponding author. Fax: +55-71-356.2155. E-mail: zilton@cpqgm.fiocruz.br Received 23 April 2001 Accepted 6 June 2001
}

vary considerably. Therefore, recovering of worms is impractical and the counting of lesions or eggs in the whole liver would be cumbersome and of doubtful value. Better approach would consist in establishing the relationship between the infective inoculum and the degree and distribution of septal fibrosis within the liver.

Thirty-five young Wistar rats of both sexes, 150$200 \mathrm{~g}$, maintained in separated cages, with free access to water and to a commercial balanced diet for rats, were divided into 7 groups of 5 animals each, according to the inocula of $C$. hepatica they received: $15,30,50,100,200,500$ and 1,000 embryonated eggs, administered in $0.5 \mathrm{ml}$ of saline by a gastric tube. The eggs were extracted from the livers of infected rats, after homogenizing in a blender, washing several times, followed by sedimentation and decantation. The immature eggs were left in a humidified Petri dish at room temperature $\left(25-27^{\circ} \mathrm{C}\right)$ for 28 days to embryonate. One animal of each group was killed on the 40th, 50th, 60th, 70th and 80th day following inoculation. Pieces of the liver were immediately fixed in Bouin's fluid and embedded in paraffin. Histological sections were stained with hematoxylin and eosin, and the picro-siriusred method for collagen. Microscopically, evidences of infection were noted in all animals. Dead, disintegrating and sometimes calcified adult worms and their viable immature eggs were found within focal lesions delimited by fibrous capsule, and exhibiting necrosis and chronic, sometimes granulomatous, inflammation. These lesions kept a linear proportion to inoculum sizes. Septal fibrosis also appeared in all animals. It was mild and focally distrib- 
uted around parasitic lesions (Fig. 1A) in the animals infected up to 50 eggs. An increase in intensity was disclosed with time. Even animals infected with as little as 15 to 30 eggs, presented a tendency of the septal fibrosis to become diffuse by the 80th day of infection. Inocula of 100 eggs or more regularly resulted in diffuse and intense septal fibrosis, which was already well defined by the 40th day of
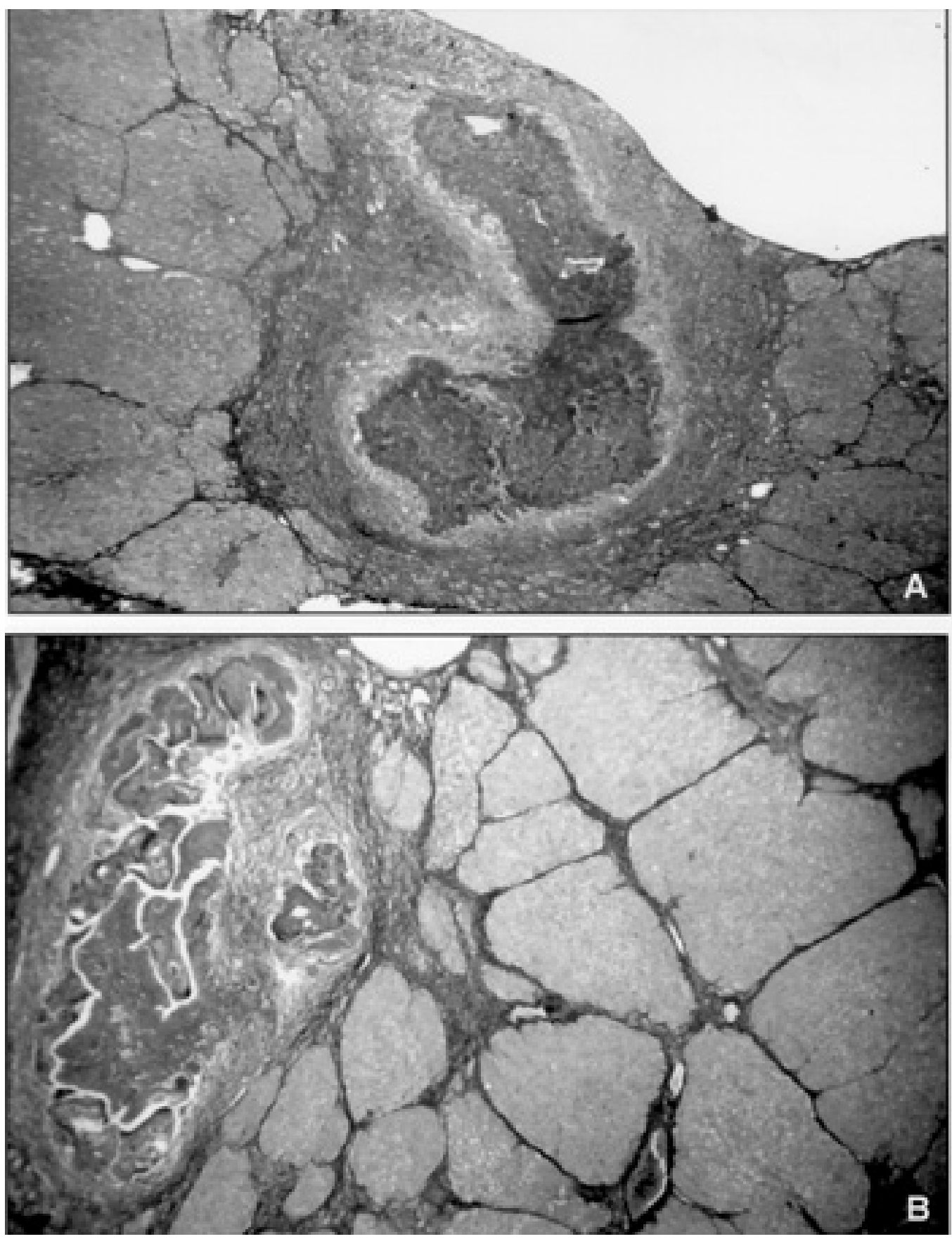

Fig. 1 - A: hepatic sub-capsular lesion, centered by a large necrotic area (dead worms), surrounded by a thick fibrous capsule, from which a few and incomplete septa sprout out toward the parenchyma in a radiated pattern. Rat infected with 15 mature eggs of Capillaria hepatica, 80th day of infection. Sirius-red stain for collagen, 120 X; B: remnants of dead worms are seen at left, within an encapsulated lesion. The liver parenchyma appears diffusely septated by fibrous bands, which sometimes isolate hepato-cellular nodules. Animal with a 40-day old infection, with 100 eggs of $C$. hepatica. Siriusred for collagen, $100 \mathrm{X}$ 
infection (Fig. 1B). With passing time the septa became somewhat thicker and branched out, but these changes were minimal. Apparently, the lesions of septal fibrosis were well defined and stabilized 40 days after infection. Higher inocula increased the severity of parasitic focal lesions, and of the septal fibrosis around them. But, the diffuse part of the fibrosis did not seem to be altered in its overall distribution and severity.

Present data are in keeping with the results already obtained with the model. Souza et al. (2000) used the $C$. hepatica model to study the effect of anti-fibrosis drugs, and also to test the antifibrosing potential of interferon- $\alpha$ (Souza et al. 2001). They used inocula of approximately 800 eggs and obtained uniform production of septal fibrosis in all experimental groups. Although septal fibrosis appears regularly when inoculum of 100 eggs is used, a working dose of 500 eggs seems to us the most appropriate. This dose is well tolerated by young adult rats. The fibrosis produced can be expected to be the same as with 100 eggs. But, as some batches may contain a variable proportion of dead and immature eggs, the now recommended inoculum would allow for not only consistent and reproducible results, but would be well tolerated, without being excessive.

\section{REFERENCES}

Andrade ZA 1991. Contribution to the study of septal fibrosis of the liver. Inter J Exper Pathol 72: 553562.
Bhunchet E, Fujieda K 1993. Capillarization and venularization of hepatic sinusoids in porcine serum-induced rat liver fibrosis: a mechanism to maintain liver blood flow. Hepatology 18: 1450-1458.

Bhunchet E, Wake K 1992. Role of mesenchymal cell population in porcine-serum-induced rat liver fibrosis Hepatology 16: 1452-1473.

Dhar A, Chawla YK 1995. Incomplete septal cirrhosis: a new entity? Trop Gastroenterol 16: 65-69.

Ferreira LA, Andrade ZA 1993. Capillaria hepatica: a cause of septal fibrosis of the liver. Mem Inst Oswaldo Cruz 88: 441-447.

Nevens F, Staessen D, Sciot R, Van Damme B, Desmet V, Cevery J, De Groote J, Van Sternbergen W 1994. Clinical aspects of incomplete septal cirrhosis in comparison with macronodular cirrhosis. Gastroenterology 106: 459-463.

Onori P, Morini S, Franchitto A, Sferra R, Alvaro D, Gaudio E 2000. Hepatic microvascular features in experimental cirrhosis: a structural and morphometric study in $\mathrm{CCl}^{4}$-treated rats. J Hepatol 33: 555 563.

Paronetto F, Popper H 1966. Chronic liver imjury induced by immunologic reactions. Cirrhosis following immunization with heterologous sera. Am J Pathol 40: 1087-1101.

Souza MM, Paraná, R, Trepo C, Barbosa Jr AA, Oliveira IR, Andrade ZA 2001. Effect of interferon- $\alpha$ on experimental septal fibrosis of the liver.' Study with a new model. Mem Inst Oswaldo Cruz 96: 343348.

Souza MM, Silva LM, Barbosa Jr AA, Oliveira IR, Paraná R, Andrade ZA 2000. Hepatic capillariasis in rats: a new model for testing antifibrotic drugs. Braz J Med Biol Res 33: 1329-1334. 
